\title{
ANÁLISIS DEL TIEMPO EN LOS ENTORNOS VIRTUALES DE FORMACIÓN
}

\section{Analysis of the time in virtual training environments}

\author{
Analyse de le temps dans les environnements virtuels \\ de formation
}

Ángel García del Dujo, Antonio Víctor MarTín García y José Manuel MuÑoz Rodríguez ${ }^{1}$ Universidad de Salamanca. Facultad de Educación. Departamento de Teoría e Historia de la Educación. c/ Paseo de Canalejas, 169. 37008 Salamanca.

Correo-e: agd@usal.es; avmg@usal.es; pepema@usal.es

Fecha de recepción: marzo de 2010

Fecha de aceptación definitiva: julio de 2010

Biblid [(1130-3743) 22, 2-2010, 111-130]

\section{RESUMEN}

El objetivo de este artículo es analizar el carácter temporal de los entornos virtuales de formación, es decir, ver si en estos entornos está o no presente el sentido, sensación, noción de tiempo; en caso positivo, se pretende, en segundo lugar, reconstruir las formas como se genera esa noción y los sentidos que adquiere en distintos entornos, es decir, identificar los mecanismos que se utilizan para generar esa noción temporal y los procesos y transformaciones que ahí

1. Los autores de este artículo pertenecen al Grupo de Investigación de Excelencia -Gr 209- de la Junta de Castilla y León (España) dirigido por el Dr. García del Dujo. El artículo presenta algunos resultados del programa de investigación que este grupo viene desarrollando en los últimos años sobre "Procesos de formación y de información en los entornos virtuales de formación". 
se producen. Asumimos aquí que, si la Red tiene algún sentido en el ámbito de la educación, además de ser un instrumento de información y un espacio de formación, es precisamente porque también ahí se mueven, conviven, relacionan y comunican, los sujetos y grupos sociales, construyendo y destruyendo, creando identidades múltiples y complejas, siendo todo ello posible solamente si su actividad responde a las coordenadas espacio-temporales tradicionales. El artículo presenta los datos de un estudio realizado en una muestra de 93 sujetos entre 20 y 30 años. Los resultados apoyan la tesis de percepción temporal y muestran diversos mecanismos de temporalización en diferentes entornos virtuales.

Palabras clave: tiempo, entornos virtuales de formación, espacios de información, espacios de formación, espacios de socialización.

\section{SUMMARY}

The objective of this article is to analyze the character of time on virtual training environments, meaning, to see whether in these environments is present or not the sense, the sensation, the notion of time; if so, it aims, secondly, to rebuilt the forms how this notion is generated and their senses on different environments, meaning, to identify the mechanisms used to generate this timing notion and the processes and transformations that are produced. We assume that, if the Network has a sense in the education field, besides being an information tool and a training space, it is also because the individuals and social groups move, coexist, have relations and communicate among them, building and destroying, creating multiple and complex identities, being all this possible only if its activity answer to timing-space traditional coordinates. The article present the data of a study conducted with a sample of 93 individuals among 20 and 30 years old. The results support the thesis of timing perception and show diverse timing mechanism in different virtual environments.

Key words: time, virtual training environments, information spaces, training spaces, socialization spaces.

\section{SOMMAIRE}

Le but de cet article est d'analyser la nature temporaire des environnements d'apprentissage virtuel, c'est-à-dire, voir si dans ces environnements est présente ou pas le sens, le sentiment, le sens du temps; dans l'affirmative, il est prévu, d'autre part, à reconstruire que des formulaires créés par cette notion et le sens qu'il faut sur des environnements différents, à savoir, identifier les mécanismes qui sont utilisés pour générer le concept temporel et les processus et les transformations qui y sont produits. Nous supposons ici que si le Réseau a un certain sens dans le domaine de l'éducation, en plus d'être un outil d'information et une zone d'entraînement, c'est précisément parce qu'il y a aussi déplacer, interagir, relation et de communiquer, les individus et les groupes sociaux, la construction et la destruction, la création d'identités multiples et complexes, avec tous les possibles uniquement si votre activité répond à la fois traditionnel coordonnées spatiales. Cet article présente les données d'une étude sur un échantillon de 93 sujets entre 20 et 30. Les résultats 
appuient la thèse de la perception du temps et de montrer les mécanismes de synchronisation différents dans différents environnements virtuels.

Mots clés: temps, environnements d'apprentissage virtuels, des espaces d'information, des espaces de formation, les lieux de socialisation.

\section{INTRODUCCIÓN: CRONOGÉNESIS DE LOS ENTORNOS VIRTUALES}

Todas las disciplinas tienen sus principios, supuestos implícitos o explícitos no cuestionados. Funcionan como coordenadas que enfocan y estructuran los fenómenos y hasta los configuran y tipifican como propios de una u otra disciplina. Pronto hará medio siglo que esta simple idea sirvió a Thomas Khun como punto de partida para trazar los mecanismos del progreso científico.

La pedagogía también tiene los suyos. Desde siempre, pero sobre todo desde que la educación se interpretó como un fenómeno susceptible de ser observado, al menos en su vertiente de interpretación pública, es un supuesto no cuestionado ni en la reflexión ni en la práctica educativa la consideración de que todo proceso educativo hace referencia implícita o explícita a una situación, es proceso situado, es decir, tiene un lugar donde acontece, que viene definido por personas implicadas y acciones que se desarrollan en alguna situación espacio-temporal.

Este principio es incuestionable en el caso de que lo educativo lleve el epíteto de social, en cuanto que la naturaleza de lo social es inevitablemente de carácter espacial y temporal (Lasén, 2000; Valencia, 2007; Zubiri, 1996), pero también es cierto que todo proceso educativo, la educación misma en un sentido constituyente, si así se nos permite expresarnos, incorpora el tiempo y el espacio como órdenes instituyentes de los propios fenómenos, no sólo como parte del escenario donde se producen y mucho menos como meros elementos del decorado, por lo que requieren de estudios e investigaciones donde estas variables tengan el protagonismo adecuado tanto en las formas de pensar dichos fenómenos y procesos como en los modos de hacer educación. El espacio y el tiempo, en nuestra interpretación y en la perspectiva que interesa a este artículo, no son los "componentes primordiales del gran telón de fondo sobre el que se pueden apreciar los fenómenos" (Valencia, 2007, 46) sino órdenes y tramas que tejen la urdimbre de cada objeto, acción y proceso; dicho en términos más sencillos, aquello que permite y hace que seamos como somos, posibilitando y limitando los procesos de configuración educativa y actuación social.

Pues bien, las nuevas tecnologías, mejor dicho, algunas interpretaciones de esta tecnología habrían puesto en entredicho este supuesto. Por eso, la pretensión última de este trabajo -y el contexto general de una parte de nuestra investigación en los últimos años- es estudiar si se produce ese carácter situado en los entornos virtuales de formación, si ese supuesto no cuestionado hasta ahora se sigue dando en dichos entornos, para lo que concretamente en este artículo nos proponemos 
analizar la presencia, sentidos, usos y comportamiento del tiempo en esos entornos; es un segundo paso y forma de averiguar si se sigue manteniendo o no ese supuesto.

Decimos segundo porque no hace mucho tiempo publicábamos un artículo (García del Dujo, 2009) con el mismo planteamiento y pretensión pero referido al espacio. Allí analizamos y comprobamos el carácter espacial de los entornos virtuales de formación, además de reconstruir las formas como se genera esa sensación, los sentidos que adquiere en los distintos entornos e incluso las transformaciones conceptuales que ahí se producen (de "espacios" a "lugares") en algunos entornos virtuales. Ahora nuestro interés y objetivos, que poco a poco iremos concretando, están en la misma dirección, pero referidos al tiempo.

Y como allí hicimos, procede que precisemos cuanto antes lo que vamos a entender aquí por entornos virtuales de formación y, sobre todo, por tiempo; lo haremos siguiendo de cerca lo que allí decíamos e introduciendo las modificaciones necesarias. Por entornos virtuales de formación entenderemos los espacios generados tecnológicamente, concretamente mediante la utilización de las nuevas tecnologías de la información y comunicación, y que son susceptibles de generar, a su vez, formación. En principio no necesitamos acotar más este concepto; si acaso reconocer que, presentado en estos términos, habría una amplia gama de entornos, dependiendo precisamente de la tecnología utilizada así como del tipo de actividad que permita esa tecnología. Esto es oportuno advertirlo, porque el comportamiento de nuestra variable bien pudiera estar en función precisamente del tipo de actividad que se esté desarrollando, ya sea productiva, de ocio, de formación..., lo que en este caso conviene tener muy presente, pues tan importante es la consideración de dichos entornos como espacios que soportan procesos de formación como una segunda interpretación en cuanto espacios susceptibles de implementar procesos de socialización, espacios en ambos casos que permiten y definen formas y mecanismos de socialización y de construcción de identidades personales y colectivas y, por ende, de formación.

Respecto del otro término, tiempo, partiremos de la noción más extendida ${ }^{2}$ como parámetro de ubicación de los fenómenos sociales, aunque ya hemos insinuado que no lo entenderemos únicamente en sentido cronológico, objetivo, de sucesión, sino como constituyente de los fenómenos y construcción social; además, en esta investigación resulta inevitable e imprescindible tomar como punto de partida esa noción porque es el significado de referencia que Castells utiliza para presentar y definir, por contraposición, el tiempo atemporal, que caracterizaría según

2. "La mayoría de los analistas sociales tratan tiempo y espacio como meros contornos de la acción y aceptan, sin advertirlo, la concepción, característica de la moderna cultura occidental, de la mensurabilidad cronológica" (Giddens, 2003, 143). 
el autor citado la temporalidad en la Red, propuesta que nosotros precisamente cuestionamos, como más adelante se verá.

Propongo la idea de que el tiempo atemporal, como he denominado a la temporalidad dominante en nuestra sociedad, se da cuando las caracteristicas de un contexto determinado, a saber, el paradigma informacional y la sociedad red, provocan una perturbación sistémica en el orden secuencial de los fenómenos realizados en ese contexto. Esta perturbación puede tomar la forma de condensar la secuencia de los fenómenos con el fin de lograr la instantaneidad, o también introducir una discontinuidad aleatoria en la secuencia. La eliminación de la secuenciación crea un tiempo indiferenciado, que es equivalente a la eternidad (Castells, 2001, 542).

La combinación de las dos acotaciones terminológicas que acabamos de hacer nos lleva a una reflexión que apunta directamente al interés de esta investigación y de la que queremos dejar constancia ya en los inicios, aunque sea en términos muy generales todavía. Interpretar el tiempo -y el espacio- de una u otra manera no es cuestión menor en ninguna sociedad, nunca lo fue en la historia ni lo es hoy tampoco. El tiempo -y el espacio- son algo más que recursos exógenos para datar y ubicar los acontecimientos, son claves de inteligibilidad social (Muntañola, 2007) que estructuran la identidad y la cultura de las personas y de los pueblos, que no son posibles, se malinterpretan y desestructuran en contextos de ausencia de tramas espacio-temporales: "...cada cultura es ante todo una determinada experiencia del tiempo y no es posible una nueva cultura sin una modificación de esa experiencia" (Agamben, 2007, 131).

No será lo mismo, para la cultura de nuestra sociedad, que en parte es informacional, y para la identidad de sus gentes, grupos y pueblos, interpretar la Red como un espacio de flujos que induce un tiempo atemporal, lo que termina en una imagen del ciberespacio como diferente y ajeno al espacio de los lugares y al tiempo de la vida cotidiana, que hacerlo en términos de numerosos y distintos espaciostiempos, órdenes diversos de espacialidad y temporalidad pero con estructuración y vertebración espacial y temporal, lo que no provoca esa "disyunción sistémica entre lo global y lo local" que aparece en el planteamiento de Castells y posibilita, por tanto, la construcción de identidades en el contínuum dentro-fuera de la Red. Sólo en este segundo caso estaríamos contribuyendo a eliminar esa especie de oxímoro -tiempo atemporal- que, junto a la aporía en la que termina la cuestión del espacio cuando se trata de procesos de formación, actúan como mecanismos de resistencia en las actitudes que algunos profesionales mantienen ante las nuevas tecnologías, concretamente en el caso de algunos usos y prácticas que cuestionan el concepto tradicional y casi espontáneo de espacio y tiempo en educación.

En publicaciones anteriores (García del Dujo, 2003; García del Dujo, Martín García y Pérez Grande, 2004; García del Dujo, Muñoz Rodríguez y Hernández Serrano, 2009) hemos apuntado a las percepciones y representaciones, actitudes y creencias, que profesionales y usuarios tenemos de las propias tecnologías, no ya del modo como se produce y en qué consiste el conocimiento, como factor a 
considerar; es decir, variables culturales -y no epistemológicas ni metodológicas, ni siquiera propiamente pedagógicas- que estarían incidiendo en la introducción y formas de uso de las nuevas tecnologías. Modos de ver e imaginar estas tecnologías derivados de una determinada concepción del espacio y tiempo y de una visión de la educación concebida en estas coordenadas y sus derivadas (el concepto de presencia como contigüidad física simultánea) que favorecerían y explicarían su uso para determinadas actividades, por ejemplo, informativas, pero no tanto para otras, más propiamente formativas y sociales.

\section{MARCO TEÓRICO DE LA INVESTIGACIÓN: CIBERESPACIO, TIEMPO Y EDUCACIÓN}

Más allá de analizar conductas normales o desviadas, propias o impropias, de personas o grupos sociales en la Red para interpretarlas desde un punto de vista socioeducativo, nos preocupa y estamos interesados, como puede apreciarse en la exposición que venimos haciendo, en conocer y valorar si los comportamientos de las personas en la Red siguen estando sujetos a las mismas variables espaciotemporales de los entornos tradicionales. Estamos aceptando que, si la Red tiene algún sentido en el amplio campo de la educación, además de ser un instrumento de información y un espacio de formación, es precisamente porque también ahí se mueven, conviven, relacionan y comunican, los sujetos y grupos sociales, construyendo y destruyendo, creando identidades múltiples y complejas, siendo todo ello posible solamente si su actividad responde a las coordenadas espacio-temporales tradicionales.

No ignoramos que a este respecto existen diferentes discursos, que podemos sintetizar en los dos siguientes: aquellos que interpretan que la Red es a-espacial y a-temporal y quienes consideran que también ahí siguen presentes las coordenadas que han hecho posible a lo largo de la historia hablar de los procesos educativos como procesos situados en un tiempo y en un espacio y del sujeto como especie con sentido y noción de espacio y tiempo, que vive territorializado y temporalizado, con sensación de espacialdad y temporalidad en cada uno de sus quehaceres independientemente del lugar donde se produzcan, que es la forma como va creando y tejiendo su identidad.

La primera posición viene a señalar que las nuevas tecnologías trascienden, alteran y cuestionan, si no anulan, los referentes espaciales y temporales tradicionales del sujeto y, en consecuencia, estaríamos ante ausencia de sentidos, usos, comportamientos y experiencias del espacio y del tiempo en la Red por parte del sujeto, o al menos serían usos y experiencias de otro espacio y otro tiempo, el llamado ciberespacio. Trasladándolo a nuestro ámbito, estas tecnologías cuestionarían el sentido situacional, espacial y temporal, de las acciones y procesos sociales y educativos que se llevan en los entornos que ellas crean. No tanto como artefactos e infraestructuras que carecen de esas propiedades cuanto, lo que es más importante para nosotros, por su capacidad de generar entornos con ausencia de la 
dimensión cronotópica propia de la especie humana, poniendo así en entredicho la interpretación de cualquier proceso acontecido en la Red como actividad social, comunicativa y relacional, integrada en el conjunto de actividades sociales en las que se ve envuelto el sujeto en la vida cotidiana.

En la denominada sociedad de redes, el llamado espacio de flujos habría sustituido al espacio cronotópico tradicional y la extensión habría dejado de ser, en beneficio de la compresión, la principal característica para estudiar individual y colectivamente el espacio y el tiempo y definir así la sociedad y la cultura. "La compresión del tiempo, que ha abolido la distancia entre los continentes, modifica nuestra relación con la cultura y con el consumo de los objetos de conocimiento" (Debray, 1991, 242).

Un nuevo paradigma cultural hecho posible por una tecnología que transforma radicalmente las dos dimensiones principales de la vida humana, el espacio y el tiempo, llegando a posibilitar el desprendimiento en los individuos de sus rasgos culturales locales, reintegrándolos en redes y espacios de flujos globales (Castells, 2001). La Red sería el soporte y producto material de cualquier práctica socioeducativa, pero sin posibilidad de proporcionarle referentes espacio-temporales. Hablar del espacio y del tiempo, desde este primer punto de vista, es hacerlo de algo, estructura, funciones, acontecimientos, que ha transformado el orden establecido en la sociedad. Los espacios y los tiempos que vienen conformando la vida social de los humanos comienzan a perder protagonismo; las comunidades dejan de controlar directamente sus espacios y tiempos de referencia y todo lo que en ellos acontece, reconvirtiendo así las formas habituales de organización y establecimiento de los espacios tradicionales de convivencia. Y en este mismo sentido, la actividad socioeducativa se desvincula y desgarra de contextos naturales, locales, con lugares y acontecimientos, presencia e inmediatez, pudiendo llegar a hablar de desmaterialización de la actividad social y educativa, reducida a "mero proceso computacional desprovisto de las tramas espaciales, temporales, objetuales y personales" (García del Dujo, 2009, 108) que configuran cualquier proceso educativo y social.

El otro discurso, donde se inserta precisamente este trabajo, interpreta esta corriente devaluadora y malinterpretadora del espacio y del tiempo como efecto de la exaltación de lo económico en detrimento de otros factores sociales y culturales. Más allá de una configuración "aespacial" y "atemporal" de la sociedad, es posible hablar de construcción de identidades personales y colectivas en los entornos virtuales en tanto que lugares y momentos, acontecimientos en la Red, siempre que los procesos de desespacialización y destemporalización promovidos por las nuevas tecnologías vayan seguidos de otros con capacidad para reespacializar y retemporalizar la actividad que ahí se desarrolle. Este cambio de perspectiva no es sólo ni siquiera el contrapunto localista a una perspectiva global de la sociedad, sino también la creencia de que, detrás de los espacios de redes -delante o al lado, pero no con estructura jerárquica-, existen espacios de lugares y ocasiones, sitios y momentos, múltiples espacios y tiempos que conforman la trama de cronotopos 
que coexisten o se suceden en la historia social de los humanos (Ramos Torre, 1999). La Red, entendida como lugares preferidos, momentos agradables, sitios evitados o acontecimientos repugnados, no sólo como contenedor de procesos y conexiones a escala planetaria, actúa como mediación en muchos de los procesos, individuales y sociales, que acontecen en el discurrir diario de una persona. La tecnología no crea un modelo socioterritorial aparte, salvo en casos de deterioro del sujeto, sino que esos "mundos" quedan integrados en el quehacer cotidiano y en su estructura espaciotemporal, como ha venido ocurriendo desde siempre.

\section{ESTRATEGIA Y DisEÑO METOdOLÓGICO, TÉCNICAS E INSTRUMENTOS}

\subsection{Objetivos}

En función del planteamiento que venimos haciendo, los objetivos de investigación en el marco de este artículo quedaron formulados en los siguientes términos:

Objetivo 1.- Analizar el carácter temporal de los entornos virtuales de formación, es decir, comprobar si en estos entornos está o no presente el sentido, sensación, percepción o noción de tiempo;

Objetivo 2.- en caso positivo, pretendemos, en segundo lugar, reconstruir las formas como se genera esa noción, es decir, identificar los mecanismos que se utilizan para generar esa noción de temporalidad (mecanismos de temporalidad en la Red: forma y mecanismos de destemporalización/ atemporalización (desequilibrio, coexistencia, instantaneidad, solapamientos...) y procesos de retemporalización (buscar un orden, movimientos, acciones, sensaciones...) y

Objetivo 3.- comprobar la existencia de diferencias significativas entre los subgrupos de la muestra en la percepción temporal en entornos virtuales de formación en función de las siguientes variables: edad, género, nivel de titulación académica, de uso y conocimiento de Internet y de competencia en el manejo de recursos y programas informáticos.

Expresado en términos de objetivo general, buscamos la deconstrucción ${ }^{3}$ del concepto de tiempo en los entornos virtuales de formación por entender que este camino puede llevar a explicar por qué se produce educación en esos entornos y a comprender cómo ese proceso de formación sigue siendo un proceso situado.

3. La deconstrucción consiste en tomar una idea, una intuición o un valor y comprender sus mecanismos quitando el cemento que la constituye. DerRida (1999). 


\subsection{Diseño de las actividades-tarea}

Se propuso a los sujetos tres situaciones-tarea diferentes que implicaban el uso de diversos recursos y actividades on line, que debían realizar en un tiempo no inferior a 40 minutos continuados.

Situación 1.- Búsqueda de información: se pidió a los sujetos que participaron en el estudio que realizaran diversas búsquedas de información (comparativa de ofertas de productos, precios de viajes y hoteles, búsqueda de documentos académicos y de diversa información educativa, etc.). Con el fin de tener una única puntuación se ha calculado la media global de los ítems que pertenecen a esta categoría: media total $=2,56$, desviación típica $=0,32$ (Vmín. = 1,47 y Vmáx. = 3,24).

Situación 2.- Actividad formativa: en este caso los sujetos valoraron los ítems del cuestionario en relación con su participación en actividades de formación on line recientemente realizadas por ellos. La media global de los ítems que pertenecen a esta categoría es $=2,66$, desviación típica $=0,37$ (Vmín. = 1,64 y Vmáx. = 3,79).

Situación 3.- Participación en redes sociales: los sujetos valoraron los ítems del cuestionario en relación con su participación en redes sociales (Facebook y/o Twitter). Con el fin de tener una única puntuación se ha calculado, al igual que en los dos casos anteriores, la media global de los ítems que pertenecen a este indicador: media total $=2,56$, desviación típica $=0,32($ Vmín. $=1,47$ y Vmáx. $=3,24)$.

\subsection{Muestra e instrumentos de recogida de datos}

Con objeto de buscar una cierta homogeneidad en el grupo de sujetos que forman parte del estudio se realizó una selección de estudiantes conforme a los siguientes criterios: intervalo de edad entre 20 y 30 años y pertenecer a alguno de los tres niveles formativos -diplomado, licenciado, máster en estudios vinculados al ámbito educativo (educación social, pedagogía, magisterio...)-; nivel de uso medio o avanzado en el manejo del ordenador así como en conocimiento de las distintas herramientas informáticas y nivel de conocimiento y uso de Internet también medio o avanzado. Conforme a estos criterios, se obtuvo una muestra válida de 93 sujetos que aparece caracterizada del modo siguiente: la media de edad de los sujetos es de 23 años, siendo el 81,7\% mujeres y el 18,3\% varones. De ellos, el 54,8\% estudian una licenciatura, el 39\% diplomatura y el 31,5\% restante máster. Respecto al nivel de conocimiento y uso del ordenador (medido a través del sumatorio de medias en 6 ítems: conocimiento y uso de sistemas operativos, procesadores de textos, tratamiento de imágenes, creación de páginas web y FTP, reproductores y/o grabadores, lenguajes de programación), se sitúa en una media de 2,69 y desviación típica de 0,44 (puntuación mínima 1,75 y máxima 3,68) (nivel usuario medio alto), siendo el conocimiento de sistemas operativos $(66,7 \%)$ y el uso de procesadores de texto $(68,8 \%)$ los de nivel de conocimiento 
más alto y el conocimiento y uso de lenguajes de programación los de más baja puntuación (una gran parte de los sujetos [40,9\%] manifiesta un conocimiento nulo o de usuario en este ítem). Por otro lado, el nivel de conocimiento de Internet se midió a través de 12 actividades diferentes (desde buscar información, comunicarse por e-mail, uso de chats, participar en redes sociales, juegos on line, plataformas de aprendizaje, compras on line, etc.). La media de este conjunto de ítems es de 2,69 , en un rango que va de 1 (no usuario) a 4 (experto).

Un aspecto central de este trabajo ha sido la elaboración y aplicación de un instrumento para valorar la percepción del tiempo en entornos virtuales. La escala fue aplicada conjuntamente con un cuestionario "ad hoc" para la medida de las variables de tipo sociodemográfico y de identificación de los sujetos. Tras la depuración y eliminación de algunos ítems, a partir de una prueba piloto y de la opinión de expertos, la escala final aplicada consta de 33 ítems (medidos desde el ítem 21 al 53 en el cuestionario). Los análisis de fiabilidad de la escala revelan un coeficiente Alfa de Cronbach = 0,925, cuya lectura es muy satisfactoria para este tipo de estudios. Por otro lado, se determinó la validez de contenido mediante la revisión bibliográfica sintetizada en el marco teórico de las investigaciones llevadas a cabo por el equipo de investigación que presenta este artículo. Por lo que se refiere a la validez de constructo, hemos optado por determinarla mediante el análisis factorial. Así, dado que el objetivo de esta escala es medir la percepción temporal de los usuarios en los espacios virtuales, procedimos a realizar los análisis factoriales pertinentes que nos sirvieron para determinar la existencia de estos contenidos en el conjunto de ítems, realizando un estudio en profundidad de los más relevantes a los objetivos del estudio.

\section{ANÁlisis y RESUltados}

\subsection{Sobre el carácter temporal de los entornos virtuales (objetivo 1)}

La medida de la percepción total del tiempo (variable que surge del sumatorio de las puntuaciones medias de las tres situaciones-tarea planteadas: media total de situación a + media total de situación b + media total de situación c) es igual a 2,60, desviación típica 3,008 (puntuación mínima 1,74 y máxima 3,46). Teniendo en cuenta que la escala de medida contempla un intervalo de 1 a 4 (sin percepción temporal, percepción temporal media, alta y muy alta), podemos concluir que los sujetos tienen un nivel medio-alto de percepción temporal, siendo la situación de participación en redes sociales la que ofrece una percepción temporal más alta (media $=2,66)$. 


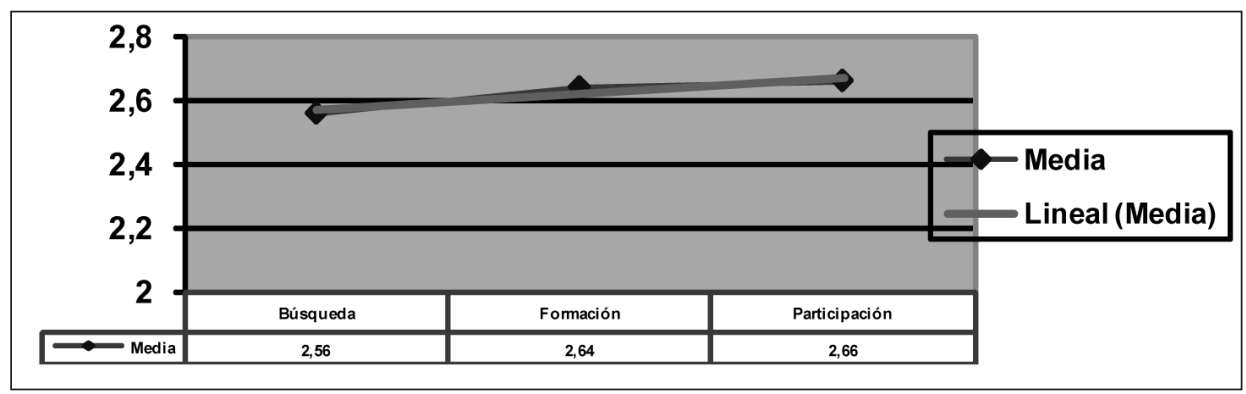

\subsection{Sobre los mecanismos de temporalidad en la Red (objetivo 2)}

Como hemos indicado, se efectuó un análisis factorial cuya matriz de componentes principales es la siguiente:

TAbla 2. Porcentaje de Varianza explicada por Cada factor RESUltante Del afaC DEL CUESTIONARIO Y VARIANZA TOTAL ${ }^{4}$

\begin{tabular}{|c|c|c|c|c|c|c|}
\hline \multirow{2}{*}{ COMPONENTE } & \multicolumn{3}{|c|}{ AUTOVALORES INICIALES } & \multicolumn{3}{|c|}{$\begin{array}{c}\text { SUMAS DE LAS SATURACIONES } \\
\text { AL CUADRADO DE LA EXTRACCIÓN }\end{array}$} \\
\hline & Total & $\begin{array}{c}\% \text { de la } \\
\text { varianza }\end{array}$ & $\%$ acumulado & Total & $\begin{array}{c}\text { \% de la } \\
\text { varianza }\end{array}$ & \% acumulado \\
\hline 1 & 5,738 & 17,388 & 17,388 & 5,738 & 17,388 & 17,388 \\
\hline 2 & 4,634 & 14,042 & 31,430 & 4,634 & 14,042 & 31,430 \\
\hline 3 & 2,395 & 7,259 & 38,688 & 2,395 & 7,259 & 38,688 \\
\hline 4 & 1,997 & 6,051 & 44,739 & 1,997 & 6,051 & 44,739 \\
\hline 5 & 1,632 & 4,946 & 49,685 & 1,632 & 4,946 & 49,685 \\
\hline 6 & 1,568 & 4,751 & 54,436 & 1,568 & 4,751 & 54,436 \\
\hline 7 & 1,431 & 4,336 & 58,772 & 1,431 & 4,336 & 58,772 \\
\hline 8 & 1,251 & 3,790 & 62,562 & 1,251 & 3,790 & 62,562 \\
\hline 9 & 1,207 & 3,659 & 66,221 & 1,207 & 3,659 & 66,221 \\
\hline 10 & 1,095 & 3,318 & 69,539 & 1,095 & 3,318 & 69,539 \\
\hline
\end{tabular}

4. Método de extracción: Análisis de Componentes Principales (se han eliminado las cargas factoriales inferiores a 0,40; el cálculo de pertinencia se realizó mediante la prueba de esfericidad de Bartlett $=1199,203$, Chi-cuadrado aproximado $=0,528$, con Significación $=0,000$ y punto de corte fijado en lambda $=1$. Medida de adecuación muestral de Kaiser-Meyer-Olkin =,625). 
Como puede apreciarse en esta tabla, los 10 factores retenidos explican el 69,54\% de la misma. Ambos datos (la varianza explicada por el primer factor y la varianza explicada por el conjunto de los primeros factores) resultan ser perfectamente asumibles en el contexto de este tipo de estudios. Por esta razón las comunalidades "explicadas" de cada una de las variables (la suma de los cuadrados de la correlación de cada variable con el conjunto de los factores retenidos) pueden considerarse, en términos generales, satisfactorias. En todo caso, y dado que los cuatro últimos factores no ofrecen datos de interés, desde el punto de vista de su aportación conceptual, a efectos expositivos describimos aquí únicamente los 6 primeros factores $(54,43 \%$ de la varianza total).

TABla 3. FACTORES EMPÍRICOS: Dimensiones DE TEMPORALIDAD EN ENTORNOS VIRTUALES

\begin{tabular}{|l|l|}
\hline \multicolumn{1}{|c|}{ DIMENSIÓN } & \multicolumn{1}{c|}{ DESCRIPCIÓN } \\
\hline $\begin{array}{l}\text { Factor I. Secuencialidad } \\
\text { temporal }\end{array}$ & $\begin{array}{l}\text { Sensación de tiempo asociada a la idea de movimiento, de ritmo, de } \\
\text { actividad, de inicio y final. }\end{array}$ \\
\hline $\begin{array}{l}\text { Factor II. Desorientación } \\
\text { temporal }\end{array}$ & $\begin{array}{l}\text { Sensación de alteración del tiempo, rupturas, desorientación, } \\
\text { acumulación y de pérdida de la noción de temporalidad. }\end{array}$ \\
\hline $\begin{array}{l}\text { Factor III. Espacialidad } \\
\text { temporal }\end{array}$ & $\begin{array}{l}\text { Sensación del tiempo asociado a la noción de normatividad social, } \\
\text { compañí, interacción social y de espacio. }\end{array}$ \\
\hline Factor IV. Linealidad & Idea de normalidad y normatividad temporal. \\
\hline Factor V. Orden temporal & Percepción de un antes y un después. \\
\hline $\begin{array}{l}\text { Factor VI. } \\
\text { Destemporalización }\end{array}$ & Sensación de provisionalidad, de tiempo efímero, atemporalización. \\
\hline Factor VI. Simultaneidad & Sensación de coincidencia, coexistencia. \\
\hline
\end{tabular}

Para realizar la selección de los ítems más representativos de las formas y mecanismos como se genera esa noción de tiempo en cada uno de los factores se ha seguido el siguiente procedimiento: en primer lugar calculamos el alfa de Cronbach para cada uno de ellos, después comparamos las medias globales entre las tres situaciones tarea -(a) Búsqueda, (b) Formación y (c) Participación- y, por último, realizamos pruebas de significación estadística en función de los segmentos muestrales. En función de esto, los ítems seleccionados más representativos son los siguientes: $23,26,29,31,38,42,44,45,49,50$ y 53, para los que se ha realizado el análisis de varianza a través del MLG (Modelo Lineal General de Medidas Repetidas $)^{5}$.

5. Los datos que permite analizar este modelo son los procedentes de un diseño con un solo grupo de sujetos y un único factor cuyos niveles se aplican a todos los sujetos. Las distintas medidas, tantas como niveles tiene el factor (tres en este caso), se toman sobre los mismos sujetos. 
- Mecanismos de organización, retemporalización y secuencialidad temporal

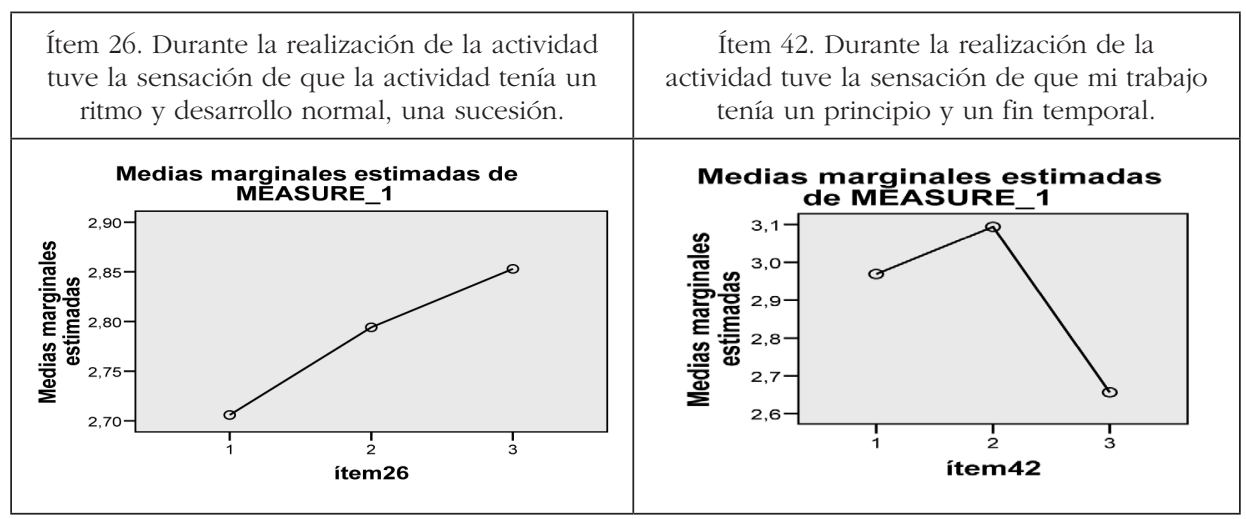

Situación-tarea: 1.-Búsqueda de información. 2.-Formación on line. 3.-Participación en red social.

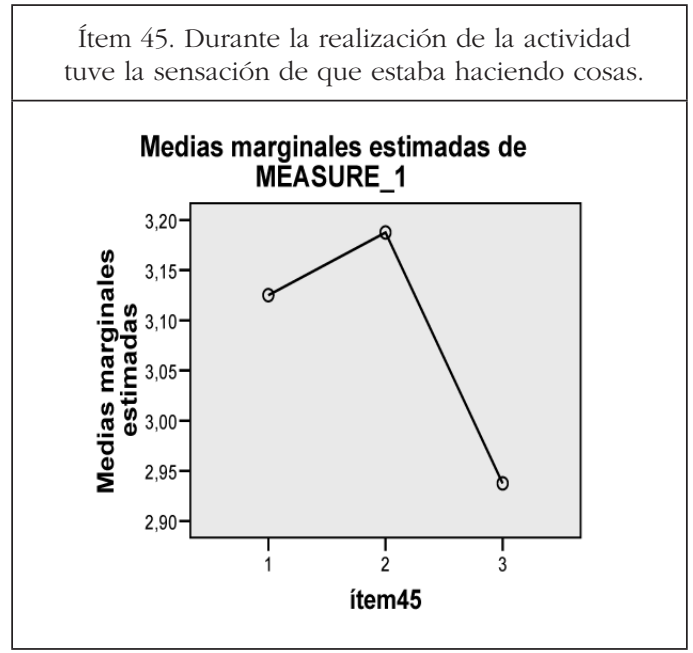

Situación-tarea: 1.-Búsqueda de información. 2.-Formación on line. 3.-Participación en red social.

En estos ítems las diferencias entre las distintas medidas del tiempo no son significativas (el nivel crítico asociado a cada uno de los estadísticos es mayor de 0,05). Esto significa que miden lo mismo en los tres momentos temporales definidos en cada una de las situaciones-tarea propuestas. Esto es, los sujetos afirman tener sensación de ritmo, desarrollo normal, sucesión, de inicio y fin en su actividad en las tres situaciones planteadas.

Del mismo modo, la percepción del tiempo que manifiestan tener los estudiantes se mide en este ítem a través de la productividad en sus acciones. No hay 
diferencias significativas en función de la búsqueda de información, formación on line, ni participación en redes sociales. Los tres grupos son conscientes del paso del tiempo en igual medida, en el transcurso de las distintas actividades planteadas.

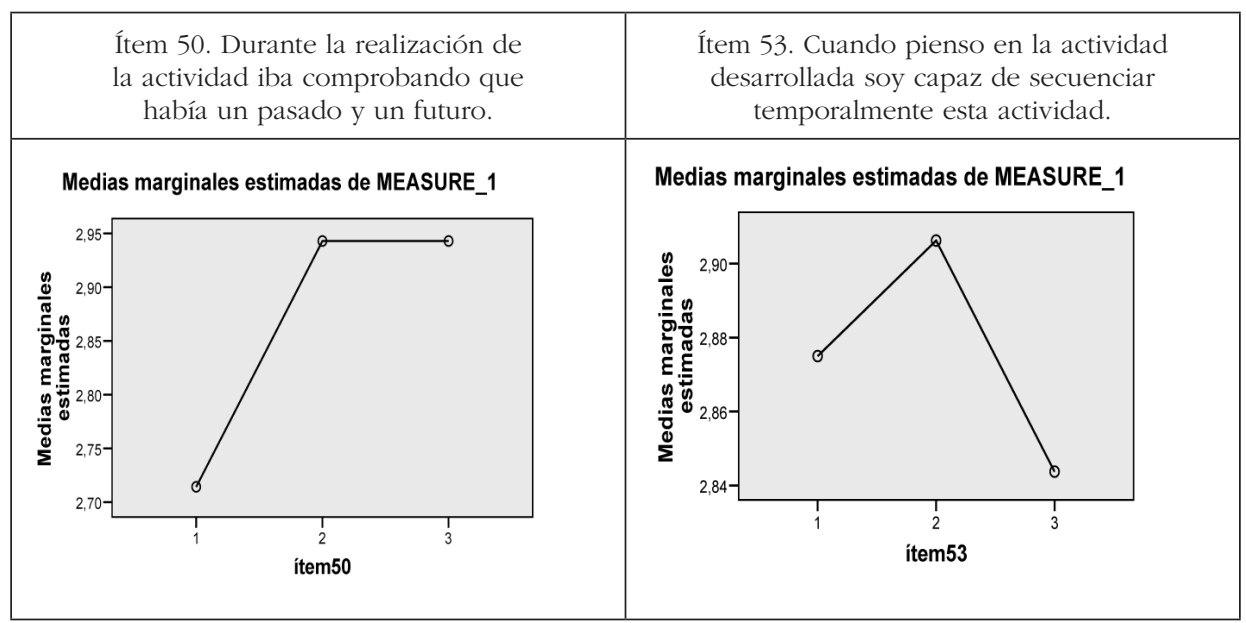

Situación-tarea: 1.-Búsqueda de información. 2.-Formación on line. 3.-Participación en red social.

En el ítem 50 las diferencias entre las distintas medidas del tiempo, en cada una de las situaciones-tarea, no son significativas. Puesto que el nivel crítico asociado a cada uno de los estadísticos es mayor de 0,05, podemos aceptar la hipótesis nula y concluimos que la sensación de pasado y futuro se da en las tres dimensiones estudiadas. Por último, el ítem 53 es el que más correlaciona con el primer factor del análisis factorial $(0,715)$. Puesto que el nivel crítico asociado a cada uno de los estadísticos es mayor de 0,05, podemos aceptar la hipótesis nula y concluimos que la percepción de la secuencia temporal es igual en las tres situaciones estudiadas. 
- Mecanismos de destemporalización

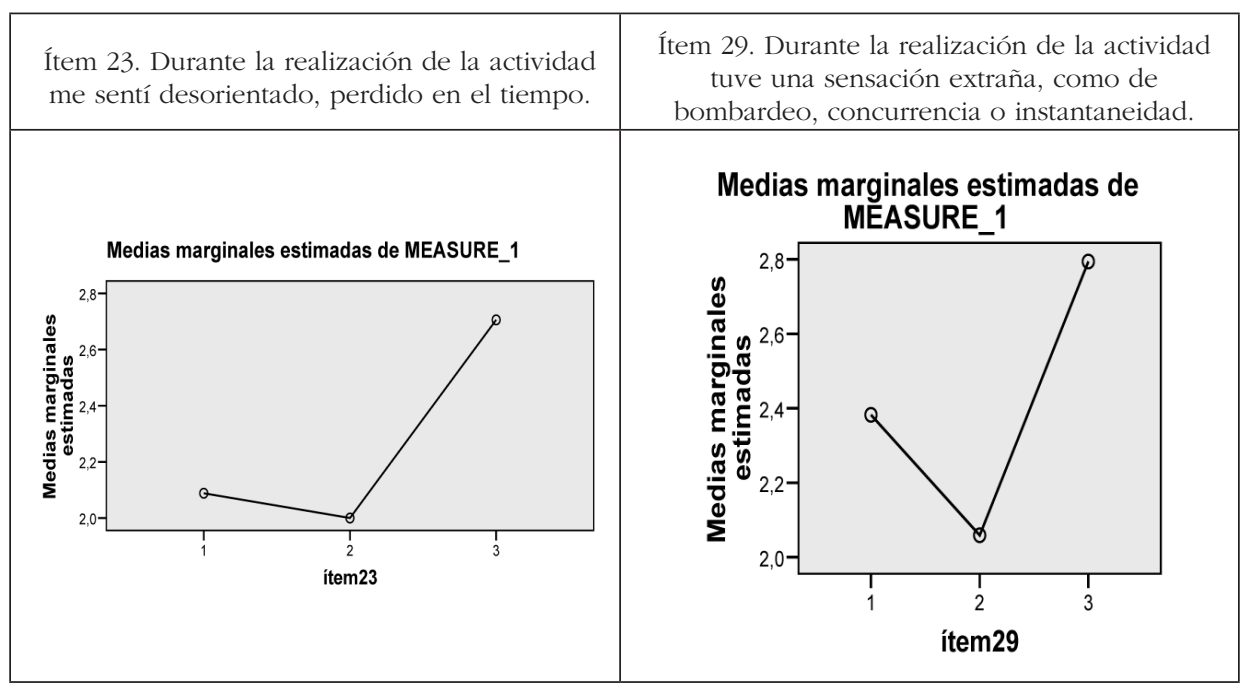

Situación-tarea: 1.-Búsqueda de información. 2.-Formación on line. 3.-Participación en red social.

En el gráfico de perfil se aprecia el efecto de los niveles del factor tiempo. Podemos observar que los sujetos tienen la sensación de estar más "perdidos en el tiempo" cuando participan en redes sociales (la diferencia de las medias es significativa al nivel ,05). Esta sensación de desorientación disminuye significativamente cuando el usuario realiza búsquedas de información o cuando realiza actividades de formación. De igual modo, la sensación de que varios momentos concurren en el tiempo es estadísticamente significativa entre los tres grupos, siendo más acusada cuando participan en redes sociales que cuando buscan información en red. Una vez más la puntuación más baja se obtiene en la escala que mide la sensación

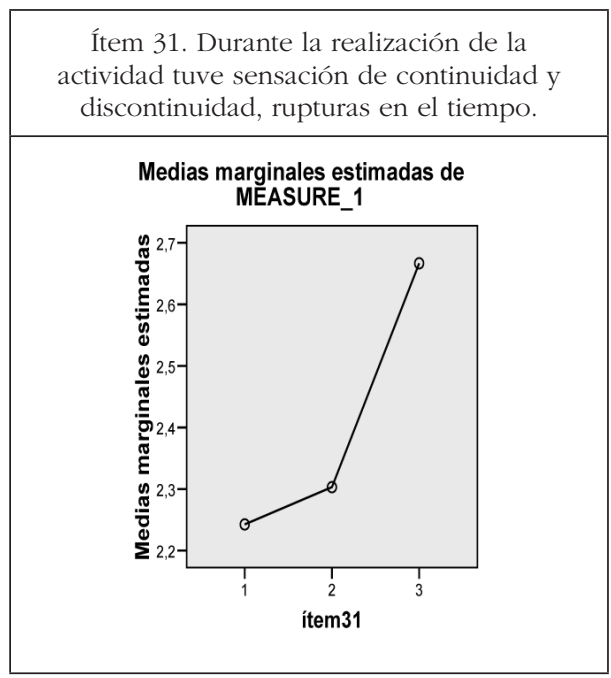

Situación-tarea: 1.-Búsqueda de información. 2.-Formación on line. 3.-Participación en red social. 
del tiempo para los alumnos que han participado en alguna actividad formativa on line.

Lo mismo ocurre en el ítem 31 para el que las diferencias entre las distintas medidas del tiempo son significativas. Los sujetos perciben la unión natural que tienen entre sí las partes del continuo del tiempo. También manifiestan percibir interrupción, suspensión, intervalos que indican rupturas en el tiempo (el nivel crítico asociado a cada uno de los estadísticos es menor de 0,05). Esta percepción del tiempo como una secuencia con intervalos es más acusada cuando los usuarios participan en redes sociales que cuando buscan información en la Red o han recibido actividades de formación on line.

- Mecanismos de normatividad, interacción y espacialidad

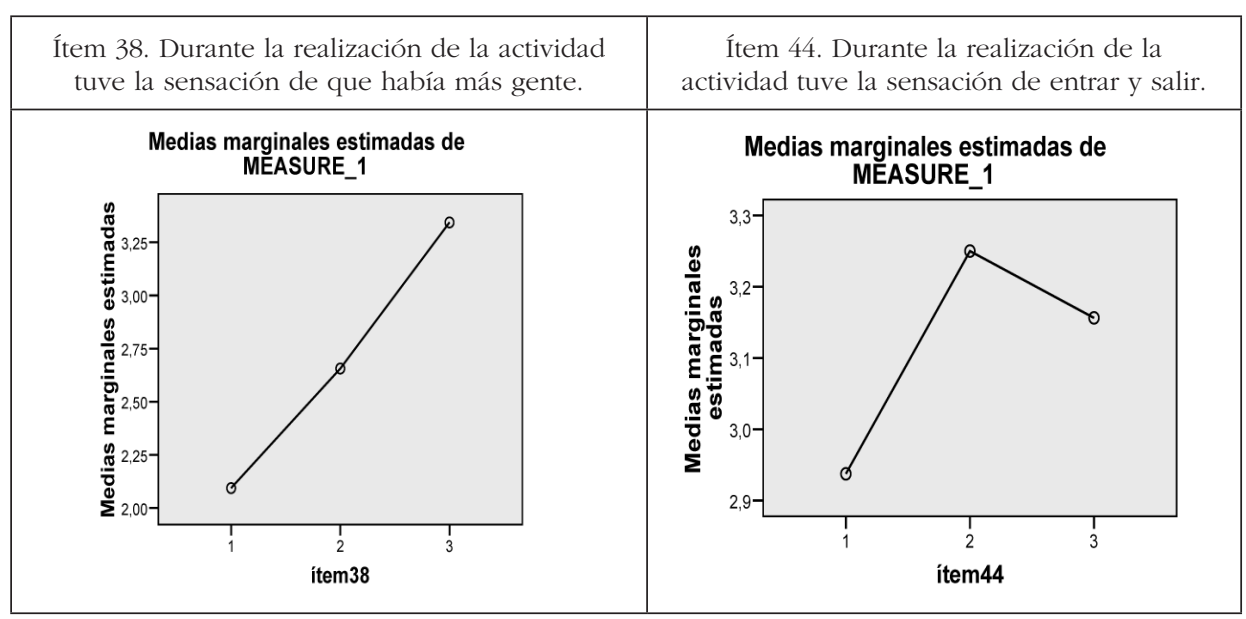

Situación-tarea: 1.-Búsqueda de información. 2.-Formación on line. 3.-Participación en red social.

La lógica del ítem 38 (Durante la realización de la actividad tuve la sensación de que había más gente) se impone en este caso. La percepción de los otros, en actividades compartidas y en situación de interacción de la red social, resulta percibida en mayor medida que en las otras dos situaciones propuestas $(p=, 000)$. En el item 44 las diferencias entre las distintas medidas del tiempo no son significativas. Los sujetos afirman tener conciencia de entrar y salir, inicio y fin. El nivel crítico asociado a cada uno de los estadísticos es mayor de 0,05 , por lo que podemos aceptar la hipótesis nula y concluir que el ítem 44 mide lo mismo en las tres situaciones planteadas. 
- Mecanismos de duración, intensidad temporal

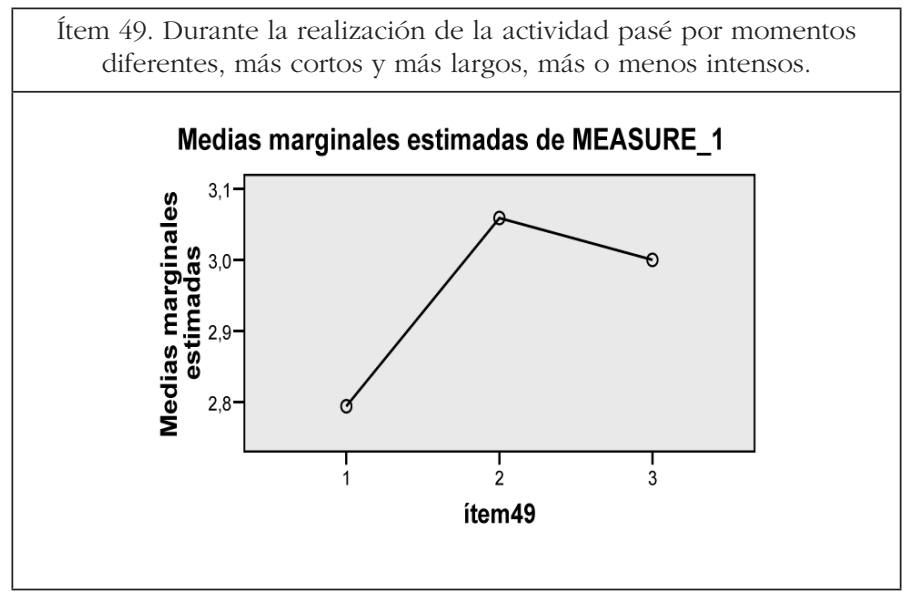

Situación-tarea: 1.-Búsqueda de información. 2.-Formación on line. 3.-Participación en red social.

Las diferencias entre las distintas medidas del tiempo son significativas. Los sujetos manifiestan tener sensaciones de duración e intensidad del tiempo (el nivel crítico asociado a cada uno de los estadísticos es menor de 0,05). En este caso, las sensaciones de permanencia, curso, lapso, período, transcurso, extensión o persistencia del tiempo medidas con este ítem son más altas en los sujetos que han recibido formación on line, seguidos de los que participan en redes sociales y por último, en los que buscan información en red.

\subsection{Sobre diferencias entre los subgrupos de la muestra (objetivo 3)}

Por último, tratamos de comprobar la existencia de diferencias significativas entre los subgrupos de la muestra en la percepción temporal en entornos virtuales de formación en función de variables como V1 (situación experimental planteada, situación-tarea), V2 (edad), V3 (género), V4 (estudios), V5 (nivel de competencia en conocimiento y uso de Internet) y V6 (nivel de conocimiento de recursos y/o programas informáticos). No se hallan diferencias estadísticamente significativas en función de las variables género y edad para los treinta y tres ítems de los que consta la prueba ni en los factores del análisis factorial. Por consiguiente, podemos afirmar que las variables género y edad no están relacionadas con la percepción temporal en entornos virtuales de formación. En cambio, en la variable estudios sí existen diferencias estadísticamente significativas (diplomado, licenciado, máster), siendo los alumnos de máster quienes manifiestan una más alta percepción temporal en las tres situaciones-tarea planteadas. 
Finalmente, comprobamos una relación directamente proporcional entre nivel de competencia en conocimiento y uso de Internet y la noción de percepción del tiempo, siendo ésta superior en aquellos estudiantes que manifiestan un nivel de uso y manejo de Internet más alto $(\mathrm{t}=-4,05, \mathrm{p}=0,000)$. Sin embargo, no aparecen diferencias significativas en función del conocimiento de recursos y/o programas de ordenador $(t=-0,926, p=0,357)$. Este resultado es sumamente interesante porque interpretamos que la percepción del tiempo no se produce por el mero hecho del manejo del ordenador, sino cuando éste se utiliza en actividades que implican el acceso a la red (Internet).

\section{CONCLUSIÓN: INTERPRETACIÓN Y DISCUSIÓN PEDAGÓGICA}

La retórica, la semántica, la ontología de la web, si así podemos expresarnos, son las del espacio, pero es imprescindible la presencia de la dimensión temporal para su correcto funcionamiento y, sobre todo, para que el sujeto no encuentre motivo alguno de resistencia en su uso. Ello no quiere decir que, dadas las características de la tecnología utilizada, tengamos que pensar en la producción y conformación de un tipo o especie particular de tiempo (el llamado tiempo atemporal) o conformarnos con una explicación hecha solamente en términos de mera traslación metafórica de la construcción-comportamiento del tiempo en los contextos tradicionales; ambas propuestas son manifiestamente insatisfactorias por su carácter paradójico, irreal, etéreo, sobre todo cuando pensamos en perspectiva educativa y de construcción de identidades individuales y sociales.

Frente a estos planteamientos, los resultados de este trabajo apuntan al hecho de que los entornos virtuales llevan consigo una noción, sensación temporal, generada en base a mecanismos y procesos análogos a los que utilizamos en los contextos tradicionales. Además, esa noción-sensación de temporalidad, a su vez, en algunos entornos virtuales da lugar a otros constructos conceptuales que vienen a coincidir con el significado de otros términos que conforman el convoy semántico del tiempo (momentos, ocasiones). Luego, no sólo presencia de noción-sensación temporal sino flujo de múltiples y distintos tiempos que el sujeto construye -temporaliza y retemporaliza- a la vez que experiencia de sensaciones de destemporalización. Además, los resultados obtenidos confirman que la forma de generar esa noción temporal y de transformar esos tiempos en momentos varía en función del uso y tipo de actividad que se realiza.

En definitiva, hemos comprobado que el tiempo en los entornos virtuales es un aspecto de la construcción social de la realidad que se activa por varios y diversos mecanismos que los sujetos ponen en marcha cuando actúan e interaccionan en la Red, lo que nos permite dimensionar la temporaliad humana en esos entornos no sólo en cuanto estructura y necesidad vital sino también como acontecimiento y accidente. Más allá del tiempo parametrizado y cronológico que de forma automática percibimos, la Red nos deja entrever ese otro tiempo distendido, fluyente, fruto de superposiciones de movimientos, de mecanismos de destemporalización 
y retemporalización, relaciones y acciones que permiten caracterizar esos entornos con potencialidad formativa.

De la misma manera que ocurría en el caso del espacio, existen formas y mecanismos que hacen visibles los modos en que los sujetos construyen, viven y significan el tiempo en la Red. Más aún, en algunos casos hay analogía entre las formas y mecanismos de construcción de la temporalidad humana en estos entornos y las que identificamos en investigaciones anteriores en relación con el espacio, lo que invita a abordar el análisis de ambas variables conjuntamente, reforzando la idea de la construcción individual y social simultánea de la unidad espacio-tiempo.

Más que hablar y estudiar por separado la problemática que subyace a la interpretación del tiempo y el espacio en los entornos virtuales, los resultados que vamos obteniendo en nuestra investigación aconsejan un estudio conjunto de ambas variables, no de forma independiente y autónoma ${ }^{6}$. En efecto, la investigación global, a la que pertenecen los resultados que aquí presentamos en torno al tiempo, se diseñó y se ha desarrollado para estudiar ambas variables de forma autónoma aunque relacionadas. Pero a medida que avanzábamos en la revisión bibliográfica, las dudas en este punto eran cada vez mayores. Nos llamaban poderosamente la atención dos cosas: por una parte, el hecho de que numerosos autores advirtiesen que resulta mucho más difícil estudiar el tiempo que el espacio, no porque sean de "naturaleza-caracteriología" diferente sino porque, mientras éste termina materializándose de una u otra manera -al menos ésa es la sensación-operación que tiene y lleva a cabo el sujeto-, aquél parece escapársele permanentemente al sujeto -al menos ésta es la sensación que todos tenemos-. Por otra parte, las dudas aumentaban a medida que encontrábamos cuestionarios, por ejemplo, sobre presencia en la web, cuyos ítems utilizan para su estudio factores, dimensiones, relacionadas con aspectos que encajan bien en la categoría de espaciales pero no recogen aspectos temporales, al menos no lo hacen en la misma cuantía y visibilidad.

Dicho de otra manera, no sería del todo desacertado estudiar el tiempo a través del espacio, en cuanto que la presencia y función del tiempo en los entornos virtuales y, en consecuencia, la manera como los sujetos experimentan en esos entornos la temporalidad sería precisamente a través de su carácter espacial, es decir, el sujeto, los sujetos, construyen los espacios-lugares de la web con presencia, intervención, uso-construcción del tiempo: ambas cosas las

6. Son muchos y diversos los autores que apuntan en esta misma dirección (RAmOs, 1999; CASTRO Nogueira, 1997, 2005; Toboso y Valencia, 2008). "Un espacio que está intensamente procesualizado no puede ser sino un espacio temporalizado. Es lógico que sea así. Es más, eso ocurría con cualquier espacio, pues poco a poco que lo pensemos como expresión y producto de prácticas reales, y aun cuando inicialmente hayamos optado por separarlo analíticamente del tiempo, acabaremos topándonos con el espacio-tiempo o, por decirlo al modo de Batjin, con los cronotopos que las realidades procesuales engendran y en las que se expresan" (RAmos, 1999, 381). 
hacen y experimentan a la vez (espacios-lugares, tiempos-momentos y ocasiones) y de la misma manera, es decir, en base a los mismos factores y dimensiones; en consecuencia, su estudio debería ser necesaria e inevitablemente simultáneo e interrelacional.

Es ésta una reflexión relevante para la investigación general que estamos llevando a cabo, pues estaríamos ante un resultado de alto interés para entender y diseñar los procesos formativos y de socialización en entornos virtuales. No es un cambio de objetivo sino de planteamiento, enfoque y procedimientos para su consecución; y, sobre todo, no es incompatible con el enfoque, planteamiento y resultados que aquí presentamos respecto del tiempo.

\section{REFERENCIAS BIBLIOGRÁFICAS}

Agamben, G. (2007) Infancia e historia. Buenos Aires, Adriana Hidalgo.

Castells, M. (2001) La era de la información. Economía, sociedad y cultura. Vol. 1 La sociedad red. Madrid, Alianza Editorial.

Castro Nogueira, L. (1997) La risa del espacio. Madrid, Tecnos.

- (2005) ¿En qué espacio habitamos realmente los hombres? Revista de Estudios Sociales, 22, 89-98.

Debray, R. (1991) Introducción a la mediología. Barcelona, Paidós.

DERRIDA, J. (1999) La deconstrucción en las fronteras de la filosofía. La retirada de la metáfora. Barcelona, Paidós.

García del Dujo, A. (2003) Pedagogy in virtual learning environments, en Méndez Vilas, A.; Mesa González, J. A. y Mesa González, J. Advances in Technology-Based Education: Toward a Knowledge-Based Society, vol. I. Badajoz, Junta de Extremadura, pp. 201-210.

- (2009) Análisis del espacio en los entornos virtuales de formación. Teoría de la Educación. Revista Interuniversitaria, 21 (1), 103-128.

García del Dujo, A.; Martín García, A. V. y Pérez Grande, M. ${ }^{a}$ D. (2004) Procesos de Formación On Line. Salamanca, Amarú Ediciones.

García del Dujo, A.; Muñoz Rodríguez, J. M. y Hernández Serrano, M. a J. (2009) Análisis e interpretación de los entornos virtuales de formación como espacio social y educativo, en AA.VV. Educación, investigación y desarrollo social. Huelva, Universidad de Huelva, 1884-1890.

GidDENS, A. (2003) La constitución de la sociedad. Bases para la teoría de la estructuración. Buenos Aires, Amorrortu.

LASÉn, A. (2000) A contratiempo. Un estudio de las temporalidades juveniles. Madrid, cIs-Siglo XXI.

Muntañola, J. (2007). Las formas del tiempo. Vol. I. Arquitectura, educación y sociedad. Badajoz: @becedario.

Ramos TorRe, R. (1999) Red, identidad, espacio y tiempo. REIS, 86, 379-386.

Toboso, M. y Valencia, G. (2008) Una representación discursiva del espacio-tiempo social. Estudios sociológicos, 76, 119-137.

Valencia García, G. (2007) Entre cronos y kairós. México, Anthropos.

Zubiri, X. (1996) Espacio, tiempo, materia. Madrid, Alianza Editorial. 